\title{
The use of intraoperative PET probe to resect metastatic melanoma
}

\author{
Grant 0 Lee ${ }^{1}$ Nicholas G Costouros ${ }^{2}$ Terry Groome ${ }^{3}$ Mohammed Kashani-Sabet ${ }^{4}$ Stanley P L Leong ${ }^{1}$ \\ ${ }^{1}$ Department of Surgery, University of California, San Francisco, San Francisco, California, USA \\ ${ }^{2}$ Department of Radiation Oncology, University of California, San Francisco, San Francisco, California, USA \\ ${ }^{3}$ University of California, San Francisco, San Francisco, California, USA \\ ${ }^{4}$ Department of Dermatology, University of California, San Francisco, San Francisco, California, USA
}

Correspondence to Stanley P L Leong, LeongSX@cpmcri.org

\section{Summary}

Two cases of metastatic melanoma resected with assistance of an intraoperative handheld positron emission tomography (PET) probe are reported. The PET probe is increasingly being used to complement findings made during surveillance monitoring. In qualified surgical candidates metastectomy may completely remove tumour burden on the patient. Two women, one 46-year-old and another 38-year-old, presented with recurrence after having initial exploration for melanoma surgical staging performed either at the University of California, San Francisco (UCSF) or at outside institutions. Combined PET/CT scans were performed preoperatively for each patient, and the use of the PET handheld probe during surgery aided the detection of the previously undetected metastases. Neither patient suffered perioperative complications.

\section{BACKGROUND}

The number of new melanoma diagnoses is expected to surpass 100000 in the USA for the year 2008. ${ }^{1}$ While the majority of cases can be treated by local wide excision and sentinel node mapping with selective sentinel lymphadenectomy, ${ }^{2}$ about $20 \%$ of the patients will require a completion regional nodal dissection because of positive sentinel lymph node(s). When patients develop isolated recurrence as detected by positron emission tomography $(\mathrm{PET}) / \mathrm{CT}$, the metastatic disease may be resected. ${ }^{3}$ Here we present two cases of recurrent regional metastatic melanoma treated with resection after using a PET probe for intraoperative detection.

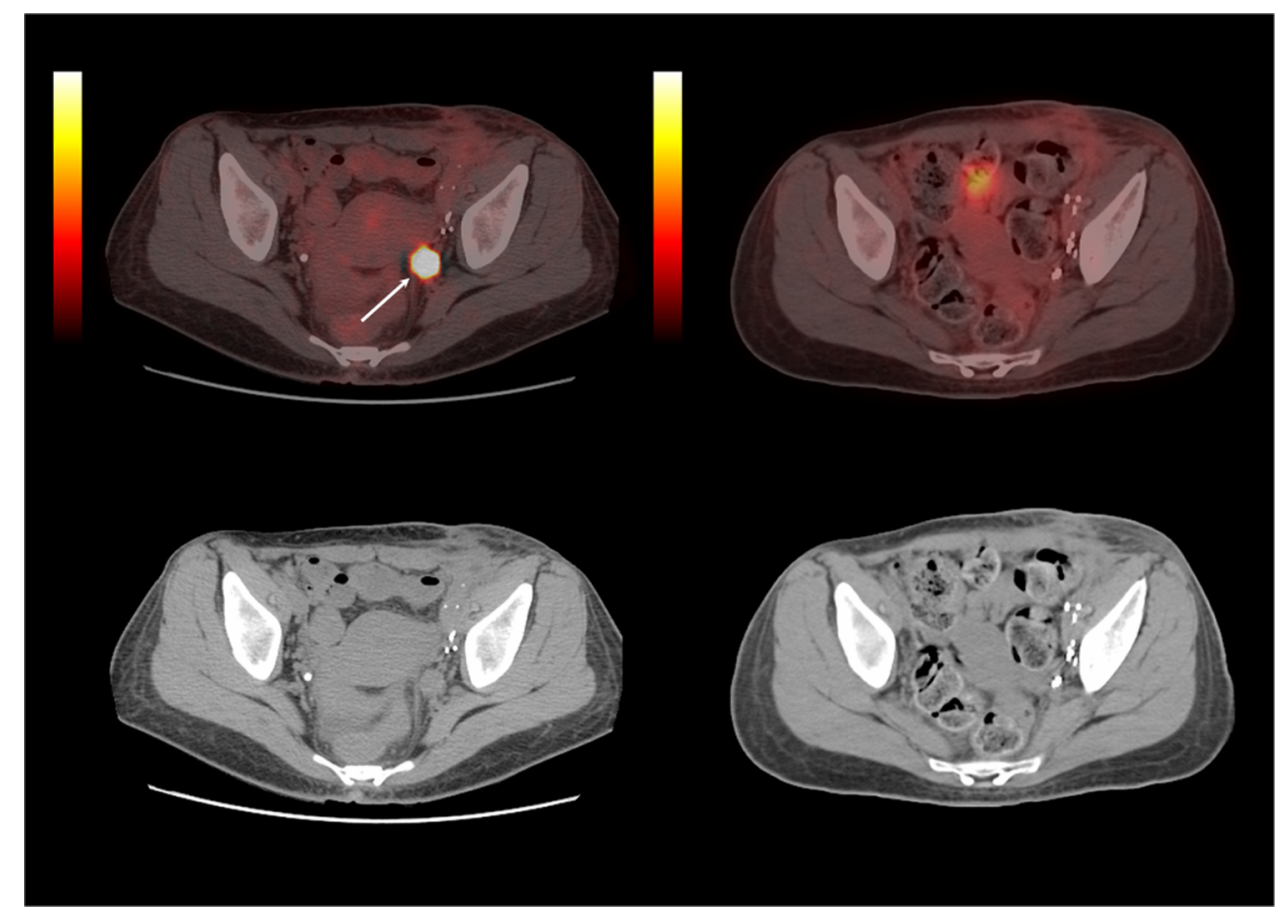

Figure 1 Study was performed on an integrated PET/CT scanner (Biograph 16; Siemens Medical Solutions) approximately 60 min following the intravenous administration of $16.6 \mathrm{mCi}$ of $18 \mathrm{~F}-\mathrm{FDG}$. Images demonstrate an FDG avid left external iliac lymph node preoperatively (left, arrow) which is no longer present postoperatively (right). 


\section{BMJ Case Reports}

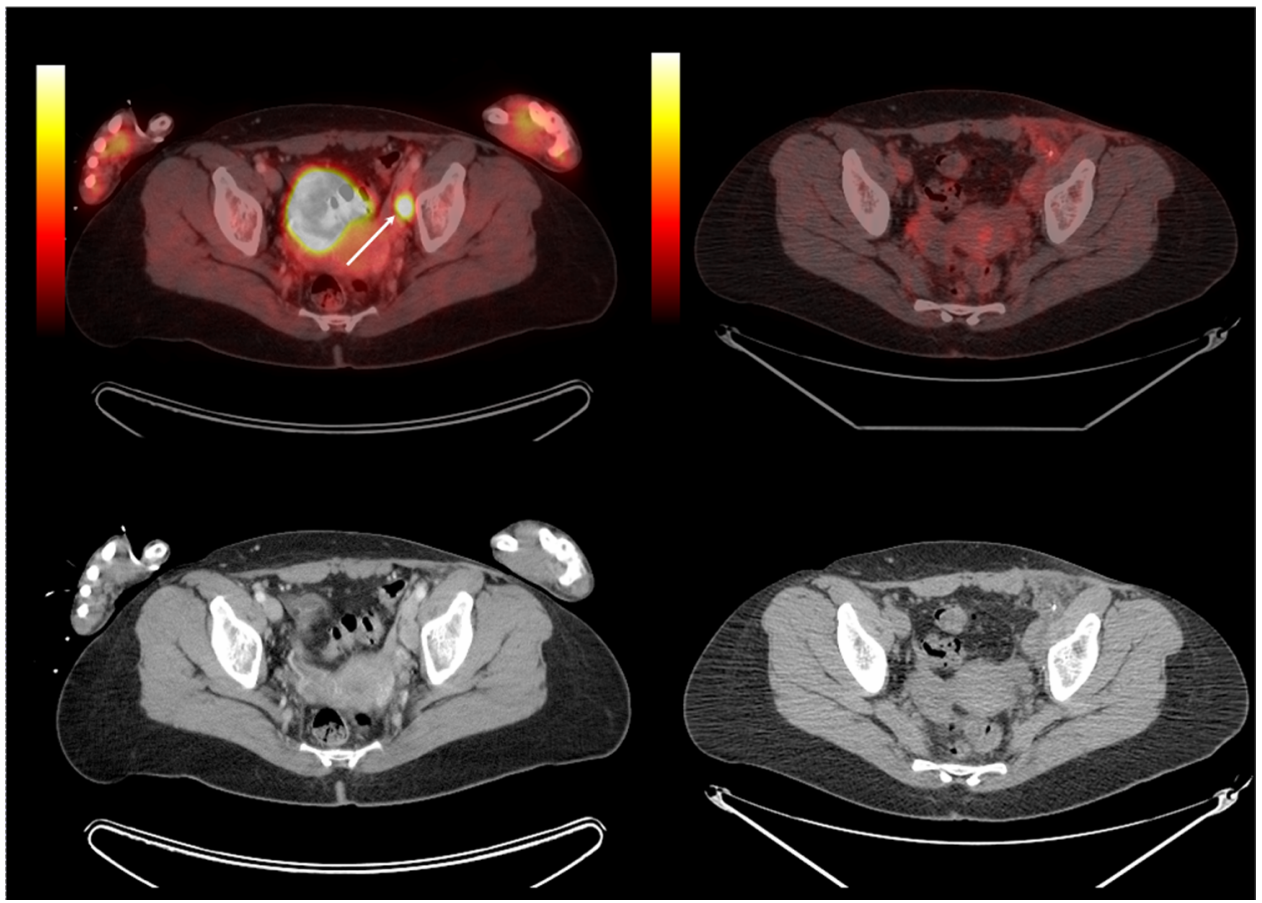

Figure 2 Study was performed on an integrated PET/CT scanner (Discovery VCT; General Electric) approximately 60 min following the intravenous administration of $11.4 \mathrm{mCi}$ of 18F-FDG. Images demonstrate an FDG avid left internal iliac lymph node preoperatively (left, arrow) which is no longer present postoperatively (right).

\section{CASE PRESENTATION}

The first patient was a 46-year-old woman who presented with haematochezia and underwent colonoscopy, which noted a $2.5 \mathrm{~cm}$ anorectal lesion positive for melanoma on pathology. She then underwent transanal excision and superficial inguinal lymph node dissection, which was positive in one out of eight lymph nodes. Radiological re-staging with whole body PET/CT and brain MRI was significant for hypermetabolic deep left iliac lymph nodes. She was advised to pursue palliative radiation treatment without surgical exploration of the deep iliac basin. The patient sought a second opinion and was seen at University of California, San Francisco (UCSF). On physical examination, there was a well-healed left-groin incision. The primary excision site at the rectum was healed. At our institution, the patient underwent deep exploration of the pelvis and resection of a mass on the left wall of the uterus which was subsequently histologically verified as a benign myoma. No resected tissue was positive for melanoma. However PET/ CT on follow-up was significant for a fluoro-2-deoxy-Dglucose (FDG)-avid lesion near the left common iliac bifurcation concerning for persistent tumour (figure 1). This lesion was not detected either grossly or by FDG injection with PET intraoperative probe during the initial surgery at UCSF.

The second patient was a 38-year-old woman who previously noted a left groin lump, which was biopsied and found positive for melanoma, at an outside institution. This was followed by a complete inguinal lymph node dissection. A surveillance PET scan detected a hypermetabolic lesion in the left pelvis. Biopsy returned positive for melanoma. A second surgical exploration was performed at the outside institution. However, the operation did not return any positive specimens. Subsequent surveillance PET scans continued to show the same lesion in the left pelvis. She was then referred to UCSF for further management. On initial presentation, she did report groin pain or genitourinary symptoms. On physical examination, her original biopsy site and groin incisions were well-healed. She did not have any palpable masses in the pelvis. Preoperatively, the patient was injected with F-18 FDG and 60 min later underwent whole body PET/CT imaging, which on review revealed an interval size increase in the FDG-avid left internal iliac lymph node compared to imaging performed 2 months earlier at the outside institution (figure 2). There was no evidence of other metastatic disease.

\section{DIFFERENTIAL DIAGNOSIS}

Regional recurrent metastatic melanoma versus lymphoma versus metastatic cancer from other primaries.

\section{TREATMENT}

The first patient underwent intraoperative PET probe identification of tumour and its resection next to the left external iliac vein. Preoperatively, the patient was injected with F-18 FDG, given intravenous fluids devoid of glucose and a urinary catheter was placed to drain the bladder. Due to previous operations the surgeons met with an extensive amount of scar tissue during exploration. However, the PET probe was able detect a $2 \mathrm{~cm}$ external iliac lymph node with a tumour: background ratio (TBR) of 4.4. The mass was resected and sent to pathology.

The second patient underwent intraoperative PET probe identification of hypermetabolic lymph nodes and their 
resection from the left external iliac and obturator nodal basins. She was given fluids devoid of glucose and had a urinary catheter placed before incision. During pelvic exploration through a new incision the tumour that was previously noted on preoperative imaging was found lying above the left obturator nerve and adhering to the pubic bone, psoas muscle and urinary bladder. A $5 \mathrm{~cm}$ mass was removed. The PET probe also detected lymph nodes in the internal iliac basin which had a TBR of 2.19 , and these were removed. The probe reading on the surgical bed following removal of detected tissue was down to background levels.

\section{OUTCOME AND FOLLOW-UP}

For the first patient, pathologic analysis of the external iliac lymph node confirmed metastatic melanoma. The patient continues on her interferon- $\alpha$ adjuvant therapy and remains disease-free at 8 months with a negative PET/CT.

For the second patient, pathologic analysis of the obturator and internal iliac lymph nodes confirmed metastatic melanoma. One month after the operation, the patient recovered well from the surgery and was treated with intravenous interferon- $\alpha$ adjuvant therapy for 1 month. She remained disease-free at 6 months follow-up, with a negative PET/CT.

\section{DISCUSSION}

Assessment for recurrence by radio-labelled F-18 FDG is gaining acceptance. ${ }^{3}$ Although radio-guided surgery was initially proposed in $1949,{ }^{4}$ it was not until 1994 when in vitro and in vivo properties of F-18 FDG probe detection was extensively described in phantom and rat models. ${ }^{5} 6$

The benefits of F-18 FDG in intraoperative probe detection include high glucose avidity for use in the anaerobic pathway in many tumour cells, in conjunction with established PET/CT imaging without requirements for additional injections, lack of a host immunological response, safe radiation burden to patient and personnel, and a short half-life of approximately $110 \mathrm{~min} .{ }^{67}$ The fluoride molecule prevents the tumour cell from metabolising the marker.

Limitations include high background glucose affinity in the brain, myocardium, kidney and bladder. ${ }^{8}$ Tumours near these organs would not provide the adequate TBR to alert the surgeon. Bladder catheterisation and diuretics are commonly used to decrease background activity in the pelvis and retroperitoneum, and ceramic composite shielding has also been proposed. ${ }^{9}$ The handheld probe also cannot differentiate inflamed tissue from malignancy. Another issue is tissue depth and sensitivity of hand-probe detection. Thin normal tissue overlying the tumour may mask a high TBR. ${ }^{10}$ Fortunately the continuing advancement of new surgical probes which combine multiple detectors attempt to minimise background radiation while increasing sensitivity. ${ }^{10}$

The use of handheld F-18 FDG detection probes have been studied in cancer patients with melanoma, ${ }^{71}$ colorectal cancer, ${ }^{11}$ non-small cell lung cancer, ${ }^{11}$ breast cancer, ${ }^{12}$ lymphoma,${ }^{13}$ iodide-negative thyroid cancer ${ }^{14}$ and ovarian cancer. ${ }^{15}$ In a retrospective review of five melanoma patients, Franc et al described the handheld PET probe having a sensitivity of $89 \%$ and specificity of $100 \%$ in detecting metastatic tissue. Three of the five patients had nonvisualised and non-palpable metastases that were detected by the probe. Numerous authors have used a TBR of 1.5 as a suggested cut-off such that the surgeon would find it comfortable enough to resect the tissue deemed as likely malignant. $^{7} 101613-15$ Other studies used a cut-off of three SDs higher than background activity ${ }^{12}$ and one study ${ }^{11}$ used a TBR of 3 .

Here we describe two patients with a previous diagnosis of melanoma and status postinguinal dissection prior to presenting at our institution. In the first case the patient had extensive scar tissue from her previous operations. The use of the PET probe guided the direction of adhesiolysis and uncovered the small $2 \mathrm{~cm}$ mass later confirmed to be malignant. The use of the probe to locate a lesion hidden under scar tissue is consistent with the conclusion of one review article on PET-probe guided surgery. ${ }^{17}$ In the second case the PET probe detected additional 'hot' lymph nodes not noted on preoperative pelvic PET imaging secondary to relatively low spatial resolution. The size and direct manipulation of the probe on the operating table offers the surgeon more information during exploration that complements the preoperative imaging. At our cutaneous oncology centre the use of the handheld PET probe is limited to certain cases of recurrent melanoma resection. A recent randomised trial on adjuvant therapy for melanoma patients who had undergone complete metastectomy did not show a survival benefit, but the researchers recognised that only select patients with a minimal number of metastasis can stand to gain any potential benefit of metastectomy combined with adjuvant therapy. ${ }^{18}$ Both patients described in this case report had regional metastasis confined to one anatomic region.

Learning points

- F-18 FDG is a commonly used marker in surveillance of recurrence or metastasis in cancer patients.

- In carefully selected individuals, the use of a handheld PET probe can aid surgeons in detecting occult lesions in the surgical bed.

- Whether metastectomy in melanoma patients confers a survival benefit remains to be elucidated.

Competing interests None.

Patient consent Obtained.

\section{REFERENCES}

1. Jemal A, Siegel R, Ward E, et al. Cancer statistics, 2008. CA Cancer J Clin 2008;58:71-96.

2. Leong SP. Sentinel lymph node mapping and selective lymphadenectomy: the standard of care for melanoma. Curr Treat Options Oncol 2004;5:185-94.

3. Shoo BA, Kangelaris G, Callen PW, et al. Detection of occult melanoma by preoperative positron emission tomography-computed tomography and intraoperative ultrasonography. J Cutan Med Surg 2010;14:130-5.

4. Yasuda S, Makuuchi H, Fujii H, et al. Evaluation of a surgical gamma probe for detection of 18F-FDG. Tokai J Exp Clin Med 2000;25:93-9.

5. Raylman RR, Wahl RL. A fiber-optically coupled positron-sensitive surgical probe. J Nucl Med 1994;35:909-13.

6. Raylman RR, Fisher SJ, Brown RS, et al. Fluorine-18-fluorodeoxyglucoseguided breast cancer surgery with a positron-sensitive probe: validation in preclinical studies. J Nucl Med 1995;36:1869-74.

7. Franc BL, Mari C, Johnson D, et al. The role of a positron- and high-energy gamma photon probe in intraoperative localization of recurrent melanoma. Clin Nucl Med 2005;30:787-91. 


\section{BMJ Case Reports}

8. Desai DC, Arnold M, Saha S, et al. Correlative whole-body FDG-PET and intraoperative gamma detection of FDG distribution in colorectal cancer. Clin Positron Imaging 2000;3:189-96.

9. Essner R, Daghighian F, Giuliano AE. Advances in FDG PET probes in surgical oncology. Cancer J 2002;8:100-8.

10. Piert M, Burian M, Meisetschlôger G, et al. Positron detection for the intraoperative localisation of cancer deposits. Eur J Nucl Med Mol Imaging 2007;34:1534-44.

11. Nwogu C, Fischer G, Tan D, et al. Radioguided detection of lymph node metastasis in non-small cell lung cancer. Ann Thorac Surg 2006;82:1815-20; discussion 1820.

12. Hall NC, Povoski SP, Murrey DA, et al. Combined approach of perioperative 18F-FDG PET/CT imaging and intraoperative 18F-FDG handheld gamma probe detection for tumor localization and verification of complete tumor resection in breast cancer. World J Surg Oncol 2007;5:143.

13. Gulec SA, Daghighian F, Essner R. PET-probe: evaluation of technical performance and clinical utility of a handheld high-energy gamma probe in oncologic surgery. Ann Surg Oncol 2006;
14. Curtet C, Carlier T, Mirallié E, et al. Prospective comparison of two gamma probes for intraoperative detection of 18F-FDG:in vitro assessment and clinical evaluation in differentiated thyroid cancer patients with iodine-negative recurrence. Eur J Nucl Med Mol Imaging 2007;34:1556-62.

15. Barranger $\mathbf{E}$, Kerrou K, Petegnief $Y$, et al. Laparoscopic resection of occult metastasis using the combination of FDG-positron emission tomography/ computed tomography image fusion with intraoperative probe guidance in a woman with recurrent ovarian cancer. Gynecol Oncol 2005;96:241-4.

16. Essner R, Hsueh EC, Haigh PI, et al. Application of an [(18)F]fluorodeoxyglucose-sensitive probe for the intraoperative detection of malignancy. J Surg Res 2001;96:120-6.

17. Gulec SA. PET probe-guided surgery. J Surg Oncol 2007;96:353-7.

18. Morton DL, Mozzillo N, Thompson JF, et al. An International, Randomized, Double-Blind, Phase 3 Study of the Specific Active Immunotherapy Agent, Onamelatucel-L (Canvaxin), Compared to Placebo as a Post-surgical Adjuvant in AJCC Stage IV Melanoma: Plenary Talk. Society of Surgical Oncology Cancer Symposium, 59th Annual Meeting, 24 March 2006, San Diego, California, USA.

This pdf has been created automatically from the final edited text and images.

Copyright 2010 BMJ Publishing Group. All rights reserved. For permission to reuse any of this content visit http://group.bmj.com/group/rights-licensing/permissions. BMJ Case Report Fellows may re-use this article for personal use and teaching without any further permission.

Please cite this article as follows (you will need to access the article online to obtain the date of publication).

Lee G0, Costouros NG, Groome T, Kashani-Sabet M, Leong SPL. The use of intraoperative PET probe to resect metastatic melanoma. BMJ Case Reports 2010; 10.1136/bcr.12.2009.2593, date of publication

Become a Fellow of BMJ Case Reports today and you can:

- Submit as many cases as you like

- Enjoy fast sympathetic peer review and rapid publication of accepted articles

Access all the published articles

- Re-use any of the published material for personal use and teaching without further permission

For information on Institutional Fellowships contact consortiasales@bmjgroup.com

Visit casereports.bmj.com for more articles like this and to become a Fellow 